\title{
Auch die Psychiatrie der Zukunft braucht Psychiaterlnnen
}

\author{
Georg Psota
}

Online publiziert: 8. Mai 2013

(C) Springer-Verlag Wien 2013

\section{Sehr geehrte Leserinnen und Leser,}

Es ist etwa 5 Jahre her, als mir das erste Mal bewusst wurde, dass sich die Fachdisziplin „Psychiatrie“ auf dem Weg zum Mangelfach befindet.

Anlass waren massive Nachbesetzungsschwierigkeiten in einem ganz bestimmten Kontext meines eigenen beruflichen Umfelds und gleichzeitig das (mir) Bekanntwerden einiger Studien zu diesem Thema. [1]

Die Studien dazu kamen vorerst aus der BRD, später dann ein Artikel aus Österreich (Katschnig [2]).

Ebenso 2010 erfuhr ich, dass die Schweizer Psychiatrie ihren (nicht geringen) Bedarf an PsychiaterInnen in beträchtlichem Ausmaß mit Psychiaterinnen und Psychiatern aus dem Ausland deckt, so habe etwa ein Drittel (!) der ungarischen PsychiaterInnen innerhalb weniger Jahre Ungarn Richtung Schweiz verlassen (was ebenso Gründe in der Schweiz, wie Folgen in Ungarn haben mag).

Im Juli 2010 wurde von der Merritt Hawkins Healthcare Company eine Studie veröffentlicht, die $\mathrm{zu}$ dem Ergebnis kam, dass der PsychiaterInnenmangel in den USA bald Krisenniveau erreichen könnte.

Sowohl im Dezember 2012 [3] als auch ganz rezent im März 2013 [4] widmen sich namhafte Kollegen im British Journal of Psychiatry im Editorial dem Thema der Attraktivität des Faches Psychiatrie für werdende ÄrztInnen und sehen in diesem Thema ein, wenn nicht das, zentrale Zukunftsthema der Psychiatrie der nächsten Jahre.

Und letztlich kommt auch die von der ÖGPB beim Institut für Höhere Studien in Auftrag gegebene und 2012 der Öffentlichkeit präsentierte Studie zum Thema

Chefarzt Prim. Dr. G. Psota $(\bowtie)$

Psychosoziale Dienste Wien, Modecenterstraße 14/B/4, 1030 Wien, Österreich

E-Mail: georg.psota@psd-wien.at
„PsychiaterInnenmangel“ für Österreich ab 2018 (also in 5 Jahren!) zu ganz ähnlichen Ergebnissen wie die zitierte USA Studie.

Und wir alle, die wir in der psychiatrischen Versorgung tätig sind, merken das bereits jetzt; manche von uns merken es ganz unmittelbar, manche nicht direkt, sondern durch Umwegswirkungen.

Was können wir daher tun, in einer Zeit, in der die Inanspruchnahme psychiatrischer Leistungen steigt und einerseits bis zu über 1 Million ÖsterreicherInnen solche Leistungen kurzfristig und zeitweilig oder wiederholt oder gar regelmäßig brauchen und auf der anderen Seite das Interesse von MedizinstudentInnen und frisch promovierten ÄrztInnen an unserem Fach zu schwinden scheint?

Wir können beispielsweise eine Form der narzisstischen Position einnehmen und meinen: wir sind eben bald die letzten edlen Vertreter unseres Faches, quasi das Syndrom „der letzten echten Mohikaner“...

Wir können eine, dem zuvor Beschriebenen recht ähnliche, Haltung einnehmen, die von workaholischer Kompensation geprägt ist: immer weniger FachkollegInnen leisten für immer mehr PatientInnen zumindest ebenso viel, quasi das Syndrom „bis zur bitteren Neige“... (um den Begriff „Burnout“ zu vermeiden)...

Auch eine depressiv-resignierende Haltung ist möglich, im Sinne von „da kann man halt nichts machen, es ist eben so, wie es ist, der Lauf der Zeit ...".

Es gibt dann noch einige weitere Möglichkeiten der Selbst-Abschaffung, z. B. die Flucht in die pure Wissenschaft, am besten ohne störende PatientInnenkontakte, gleichsam Sublimierung auf einem bestimmten Niveau...

Alle diese Formen der Einstellung kann ich nicht empfehlen, zu sehr ähneln sie neurotischen Abwehrmechanismen (wenngleich es die Neurose ja angeblich nicht mehr geben soll) und jedenfalls sind sie von geringer Konstruktivität. 
Sondern ich erlaube mir 5 Thesen:

1. Ein Mangel an Interesse PsychiaterIn zu werden ist ein bearbeitbares Phänomen und kein Naturgesetz.

2. Psychiatrie und Psychotherapeutische Medizin ist ein hoch interessantes Fachgebiet der Medizin, im 21. Jahrhundert vielleicht sogar das mit der größten gesellschaftlichen Bedeutung in Europa (und daher auch in Österreich) und allein deshalb zweifelsfrei zukunftsträchtig. Alle WHO-Daten betreffend Krankheitslast zeigen das eindrucksvoll [5].

3. Die Psychiatrie in Österreich braucht eine beträchtlichere Präsenz sowohl im Medizinstudium, als auch in der postpromotionellen Ausbildung. Hierzu ist nicht mehr vom Gleichen, sondern eine attraktive Vermittlung von Inhalten und deren Bedeutung dringend notwendig.

4. Es braucht dazu weiters, und auch generell, einen intensiven Diskurs innerhalb der Psychiatrie und eine ebenso intensiv geeinigte Darstellung nach außen.

5. Öffentlichkeitsarbeit ist kein Luxus, sondern eine unbedingte Notwendigkeit.

Wenn es uns darüber hinaus in der Folge gemeinsam gelingt, die Gestaltung der Ausbildungs- und Arbeitsbedingungen, auch in ihren Zeitabläufen, in ihren Handlungsweisen, der Zeit in der wir handeln und der Generation zukünftiger PsychiaterInnen entsprechend $\mathrm{zu}$ formulieren, dann sind wir einen meines Erachtens enormen Schritt weiter am Weg. Es sind mehr Mühen der Ebenen als Mühen der Berge und sie müssen dennoch gegangen werden.

Überdies: Man braucht uns vielerorts, aber nicht immer sind die Orte des Bedarfs die klassischen Orte des Angebotes oder der Ausbildung.
In einigen wenigen Gegenden Österreichs - und auch da auf bestimmte Abteilungen konzentriert - gibt es ausreichend geeignete BewerberInnen für nachzubesetzende Stellen und gerade diese raren Beispiele sind wichtig, denn sie zeigen, dass die Nachfrage zur Ausbildung im Fach Psychiatrie und psychotherapeutische Medizin auch prosperierend sein kann.

Wie wäre es, die LeiterInnen dieser Abteilungen zu fragen, woran es liegt, dass ihnen dergleichen gelingt?

Es ist mir wohl bewusst, dass das großteils allgemeine Formeln sind, aber so mancher Matrix helfen unspezifische Reize. Spezifischere müssen und werden folgen und brauchen die Kreativität und die Unterstützung vieler, am besten aller, österreichischen PsychiaterInnen.

\section{Literatur}

1. Symposium „Psychiatrie ohne Ärzte“ (in Kooperation mit der SGPP und ÖGPP) im Rahmen der Jahresversammlung der Deutschen Gesellschaft für Psychiatrie, Psychotherapie und Nervenheilkunde (DGPPN), 26. November 2009, Berlin.

2. Katschnig H. Are psychiatritis an endangered species? Observations on internal and external challenges to the profession. World Psychiatry. 2010;9:21-8.

3. Kleinman A. Rebalancing academic psychiatry: why it needs to happen - and soon. Br J Psychiatry. 2012;201:421-2.

4. Davies T. Recruitment into psychiatry: quantitative myths and qualitative challenges. Br J Psychiatry. 2013;202:163-5.

5. Mathers CD, Loncar D. Projections of Global Mortality and Burden of Disease from 2002 to 2030. PLoS Med 2006:3(11):e442 